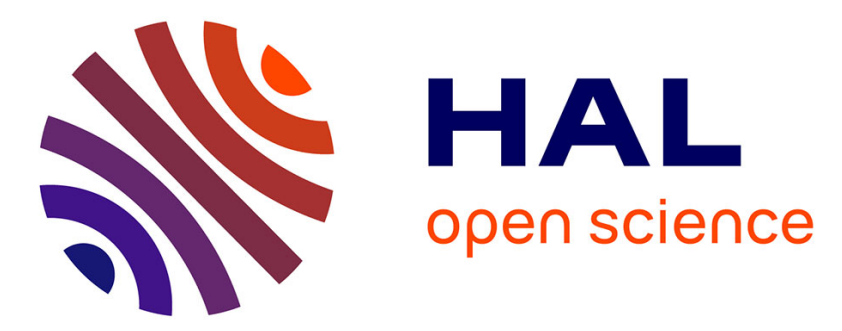

\title{
Application of a Nonlinear Modal Instability Approach to Brake Systems
}

Jean-Jacques Sinou, Fabrice Thouverez, Louis Jézéquel

\section{To cite this version:}

Jean-Jacques Sinou, Fabrice Thouverez, Louis Jézéquel. Application of a Nonlinear Modal Instability Approach to Brake Systems. Journal of Vibration and Acoustics, 2004, 126 (1), pp.101-107. 10.1115/1.1596555 . hal-00216082

\section{HAL Id: hal-00216082 \\ https://hal.science/hal-00216082}

Submitted on 30 May 2018

HAL is a multi-disciplinary open access archive for the deposit and dissemination of scientific research documents, whether they are published or not. The documents may come from teaching and research institutions in France or abroad, or from public or private research centers.
L'archive ouverte pluridisciplinaire HAL, est destinée au dépôt et à la diffusion de documents scientifiques de niveau recherche, publiés ou non, émanant des établissements d'enseignement et de recherche français ou étrangers, des laboratoires publics ou privés. 


\title{
Application of a Nonlinear Modal Instability Approach to Brake Systems
}

\author{
Jean-Jacques Sinou , Fabrice Thouverez and Louis Jézéquel
}

\author{
Laboratoire de Tribologie et Dynamique des Systèmes, Equipe Dynamique des Structures et des \\ Systèmes, Ecole Centrale de Lyon, 69134 Ecully Cedex, France
}

\begin{abstract}
A method, called the Complex NonLinear Modal Analysis (CNLMA), is proposed for the calculation of the periodic solutions of nonlinear mechanical systems with continued nonlinearities. The equivalent linearization concept and the notion of nonlinear complex modes are applied in order to analyze the steady-state responses of autonomous nonlinear systems. The CNLM-Analysis appears very interesting in regard to computational time; it also necessitates very few computer resources. This method was applied to study insta-bility phenomena in a nonlinear model with a constant brake friction coefficient.
\end{abstract}

\section{Introduction}

In recent years, nonlinear vibration phenomena have been receiving increasing attention. Parametric and nonparametric techniques have been intensively studied by many authors. Generally speaking, the nonlinear problem can be solved by techniques such as the harmonic balance method (Pierre, Ferri and Dowell [1], Cameron and Griffin [2]), the perturbation methods (Nayfeh and Mook [3]), the Galerkin's method, or numerical integration. Recently, the reduction and simplification of nonlinear systems have been studied by different strategies: the center manifold approach and normal form approach (Nayfeh and Balachandran [4], Guckhenheimer and Holmes [5], Jézéquel and Lamarque [6], Yu [7], and Sinou, Thouverez and Jézéquel [8]), the Padé approximants (Baker and Graves-Morris [9] and Brezinski [10], and Sinou, Thouverez and Jézéquel [8]) have been used as simplification methods in many studies. These techniques allow to obtain a simplified system, without loosing the dynamics of the original system, as well as the contributions of non-linear terms. Using these techniques, one has a reduction, simplification, or approximation of the original non-linear system. However, if these techniques have been applied in scientific areas such as engineering, these procedures may have problems of mathematical complexity, storage requirements, and extreme computation time.

In order to avoid these difficulties, a new method for the calculation of the periodic solutions of nonlinear systems is proposed (Boudot [11-12], and Sinou [13]). It is based on the notion of the equivalent linearization concept, and it introduces the notion of nonlinear complex modes. In recent years, many studies describe the notion of the nonlinear normal mode and the nonlinear natural frequencies associated (Rosenberg [14-15], SzemplinskaStupnicka [16-17], Shaw and Pierre [18]).

The equivalent linearization concept, as proposed by Iwan [19], can be applied to nonlinear autonomous systems in order to obtain nonlinear modal parameters. This method is based on the minimization of the difference between the nonlinear dynamic system and its equivalent linear dynamic system. The procedure of the CNLM-Analysis has been tested in the case of autonomous system that may exhibit dynamic as well as static instabilities.

This paper presents the application of the CNLM-Analysis for a brake noise vibration. Firstly, some basic concepts of friction will be introduced, and a model for the analysis of grabbing in automobile braking systems will be presented. Then, results from sta-

Contributed by the Technical Committee on Vibration and Sound for publication in the JOURNAL OF VIBRATION AND ACOUSTICS. Manuscript received July 2002; Revised January 2003. Associate Editor: A. F. Vakakis. bility analysis will be presented. The stability analysis will be performed by determining the eigenvalues of the jacobian matrix of the linearized system at the equilibrium point. A parametric study with linear stability theory will be conducted in order to determine the effect of system parameters on stability. Next, the Complex Nonlinear Modal Analysis (CNLMA) will be described. This method will be used in order to obtain the limit cycle of the non-linear system and to study the behavior of the system in the unstable region. Results from the Complex Nonlinear Modal Analysis will be compared with results obtained by integrating the full original system in order to validate the CNLM-Analysis. Finally, parametric studies using the CNLM-Analysis will be conducted in order to determine the effect of system parameters on the evolution of the limit cycle oscillations.

\section{Background}

Brake systems are often susceptible to friction-induced selfexcited vibrations. Under certain conditions, the steady state sliding motion becomes unstable and small perturbations grow with time. Due to the non-linearities, the perturbation growth is limited and the limit cycle oscillations appear. It is desirable to understand the mechanisms that cause friction-induced self-excited vibrations in order to prevent their occurrence. A greater concentration of work on the brake noise and vibrations appeared during the last years and different types of self-excited vibrations have been reported in the literature. There is no unique mathematical model and theory in order to explain the mechanisms and dynamic phenomena associated with friction. Effectively, there are different mechanisms that can excite these vibrations. According to Ibrahim [20], Oden and Martins [21], Crolla and Lang [22], the different mechanisms proposed in the literature as responsible for frictioninduced vibrations fall into four classes: stick-slip, variable dynamic friction coefficient, sprag-slip (Spurr [23]), and coupling mechanism. The first two approaches rely on changes in the friction coefficient with relative sliding speed affecting the system stability. The last two approaches use kinematic constraints and modal coupling in order to develop the instability; in these cases, instability can occur with a constant brake friction coefficient.

\section{Analytical Model}

In a previous work [8], one presented heavy trucks grabbing. In order to find design recommendations for brakes avoiding grabbing vibration, it is necessary to work with mechanical model displaying design features of a brake.

According to experimental investigations, the dynamic characteristics of the whole front axle assembly is concerned, even if the 


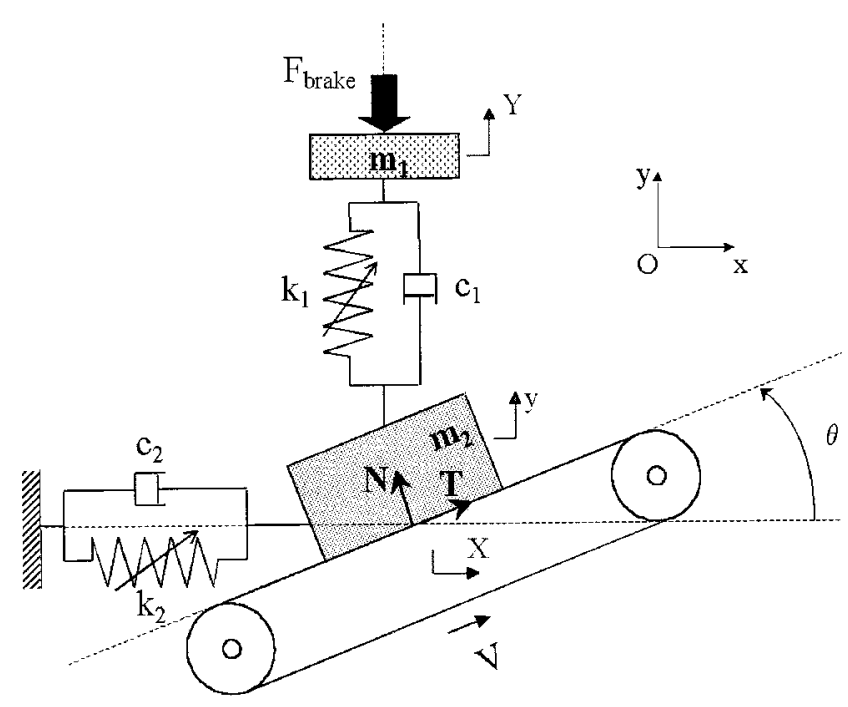

Fig. 1 Dynamic model of braking system

source of grabbing is located in the braking system. Moreover there is only a very small variation of the brake friction coefficient during a grabbing vibration event, as described by Boudot [12]. So the variation of the brake friction coefficient can be assumed to be negligible in this case, although this is not always the case for modelling brake systems. This context is selected because it is complex, both in order to be qualitatively predictive, and simple in order to allow sensitivity analysis. In this study, the mechanism used in order to explain the grabbing is a classical mechanism: brake judder is modelled as a flutter instability due to the nonconservative aspect of Coulomb's friction. The dynamic system is defined in Fig. 1. One assumes that the brake friction coefficient $\mu$ is constant, and that the tangential force $T$ is generated by the brake friction coefficient $\mu$, considering the Coulomb's friction law $T=\mu . N$.

Grabbing vibration results from coupling between the torsional mode $\left(k_{2}, m_{2}\right)$ of the front axle and the normal mode $\left(k_{1}, m_{1}\right)$ of the brake control. An important feature of this mechanism is the angle $\theta$ between the resulting force at the friction contact and the normal direction of the sliding surface. This angle can be defined by geometrical means and can also appear due to geometrical faults. In order to simulate braking system placed crosswise due to overhanging caused by static force effect, one has to consider the moving belt slopes with an angle $\theta$. This mechanism is based on dynamic coupling due to buttressing motion. This slope couples the normal and tangential degree-of-freedom induced by the brake friction coefficient: effectively, the direction of motion of the mass $m_{2}$ is indicated by this angle to the disk surface, and the two components are constraint to remain in contact and are coupled together by reaction force $N$ and friction force $T=\mu N$, where $\mu$ is the friction coefficient.

The braking force $F_{\text {brake }}$ transits through the braking command, that has a nonlinear behavior. Therefore, we consider the possibility of having a nonlinear contribution. Then, one can express this nonlinear stiffness as a quadratic and cubic polynomial in the relative displacement:

$$
\begin{aligned}
& k_{1}=k_{11}+k_{12} \cdot \Delta+k_{13} \cdot \Delta^{2} \\
& k_{2}=k_{21}+k_{22} \cdot \delta+k_{23} \cdot \delta^{2}
\end{aligned}
$$

where $\Delta$ is the relative displacement between the normal displacement in the y-direction of the mass $m_{1}$ and the mass $m_{2}$ (one has $\Delta=y-Y$ ), and $\delta$ the transnational displacement defined by the frictional x-direction of the mass $m_{2}$ (one has $\delta=X$ ). The nonlinear dynamic behavior of the brake command of the system $\left(k_{1}, m_{1}\right)$, and the non-linear dynamic behavior of the front axle assembly and the suspension $\left(k_{2}, m_{2}\right)$ are concerned, respectively.

The three equations of motion can be expressed as:

$$
\left\{\begin{array}{l}
m_{1} \ddot{Y}+c_{1}(\dot{Y}-\dot{y})+k_{11}(Y-y)+k_{12}(Y-y)^{2}+k_{13}(Y-y)^{3} \\
\quad=-F_{\text {brake }} \\
m_{2} \ddot{X}+c_{2} \dot{X}+k_{21} X+k_{22} X^{2}+k_{23} X^{3}=-N \sin \theta+T \cos \theta \\
m_{2} \ddot{y}+c_{1}(\dot{y}-\dot{Y})+k_{11}(y-Y)+k_{12}(y-Y)^{2}+k_{13}(y-Y)^{3} \\
\quad=N \cos \theta+T \sin \theta
\end{array}\right.
$$

Using the transformations $y=X \tan \theta$ and $\mathbf{x}=\{X Y\}^{T}$, and considering the Coulomb's friction law $T=\mu . N$, the nonlinear 2-degrees-of-freedom system has the form

$$
\mathbf{M .} \ddot{\mathbf{x}}+\mathbf{C .} \dot{\mathbf{x}}+\mathbf{K} . \mathbf{x}=\mathbf{F}+\mathbf{F}_{\mathrm{NL}}
$$

where $\ddot{\mathbf{x}}, \dot{\mathbf{x}}$ and $\mathbf{x}$ are the acceleration, velocity, and displacement response 2-dimensional vectors of the degrees-of-freedom, respectively. $\mathbf{M}$ is the mass matrix, $\mathbf{C}$ is the damping matrix and $\mathbf{K}$ is the stiffness matrix. $\mathbf{F}$ is the vector force due to brake command and $\mathbf{F}_{\mathbf{N L}}$ contains moreover the non-linear stiffness terms. The values of the parameters are given in Appendix A. The values of these matrices and vectors are given Appendix B.

The general form of the equations can be expressed in the following way:

$$
\begin{aligned}
\mathbf{M .} \ddot{\mathbf{x}}+\mathbf{C . \dot { \mathbf { x } }}+\mathbf{K} \cdot \mathbf{x}= & \mathbf{F}+\sum_{i=1}^{2} \sum_{j=1}^{2} \mathbf{f}_{(\mathbf{2})}^{\mathrm{ij}} \cdot x_{i} \cdot x_{j} \\
& +\sum_{i=1}^{2} \sum_{j=1}^{2} \sum_{k=1}^{2} \mathbf{f}_{(3)}^{\mathbf{i j k}} \cdot x_{i} \cdot x_{j} \cdot x_{k}
\end{aligned}
$$

where $\mathbf{f}_{(\mathbf{2})}^{\mathrm{ij}}$ and $\mathbf{f}_{(\mathbf{3})}^{\mathrm{ij} \mathbf{k}}$ are the vectors of quadratic and cubic nonlinear terms of $\mathbf{F}_{\mathrm{NL}}$, respectively. $\mathbf{M}, \mathbf{C}$ and $\mathbf{K}$ are $2 \times 2$ matrices.

\section{Stability Analysis}

The study can be divided in two parts. The first one concerns the static problem: the steady state operating point for the full set of nonlinear equations is obtained by solving them at the equilibrium point. Stability is investigated by calculating the Jacobian of the system at the equilibrium point. The second step is the estimation of the limit cycle. The nonlinear dynamic equations can be integrated numerically in order to obtain a time-history response and the limit cycle. However this procedure is too much time consuming. So one proposes a new approach, called the Complex Nonlinear Modal Analysis (CNLMA) in order to obtain the limit cycle (Boudot [11-12], Sinou [13]).

The equilibrium point $\mathbf{x}_{\mathbf{0}}$ of Eq. (4) is obtained by solving the nonlinear static equations for a given net brake hydraulic pressure. This equilibrium point satisfies the following conditions:

$$
\mathbf{K} . \mathbf{x}_{\mathbf{0}}=\mathbf{F}+\mathbf{F}_{\mathrm{NL}}\left(\mathbf{x}_{\mathbf{0}}\right)
$$

One notes that there can be more than one steady-state operating point at a given net brake hydraulic pressure, since the sprag-slip equations are nonlinear.

The stability is investigated by calculating the Jacobian of the system at the equilibrium points. The eigenvalues of the constant matrix $\mathbf{J}$ provide information about the local stability of the equilibrium point $\mathbf{x}_{\mathbf{0}}$. If all eigenvalues of the Jacobian matrix have a negative real part, the system is stable and one does not have vibration. If one root has a positive real part, one has an unstable root and vibration. The imaginary part of this root represents the frequency of the unstable mode.

Using the base parameters defined previously, the computations are conducted with respect to the brake friction coefficient. The Hopf bifurcation point is detected for $\mu_{0}=0,204$.

A representation of the evolution of frequencies and the associated real parts against brake friction coefficient are plotted in 


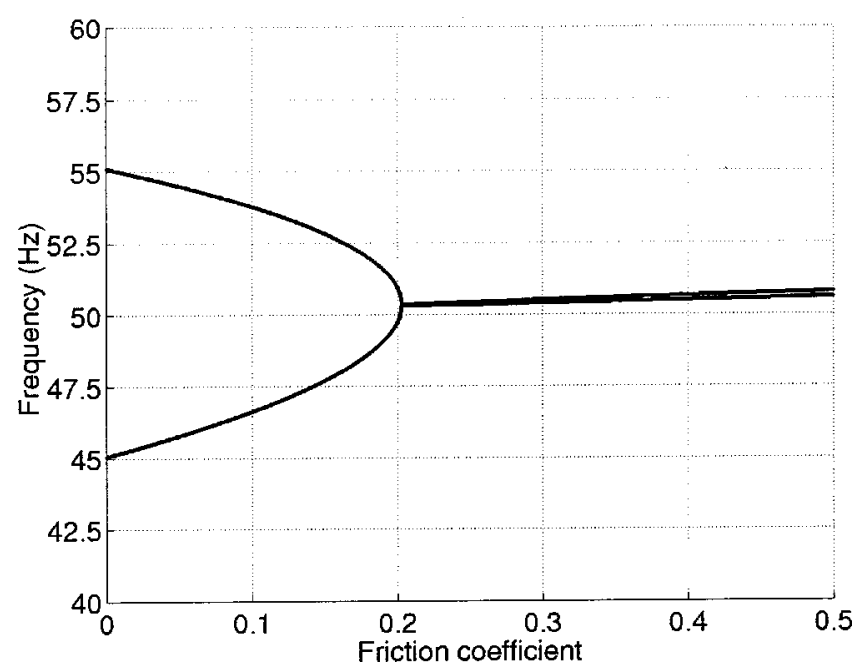

Fig. 2 Coupling of two eigenvalues

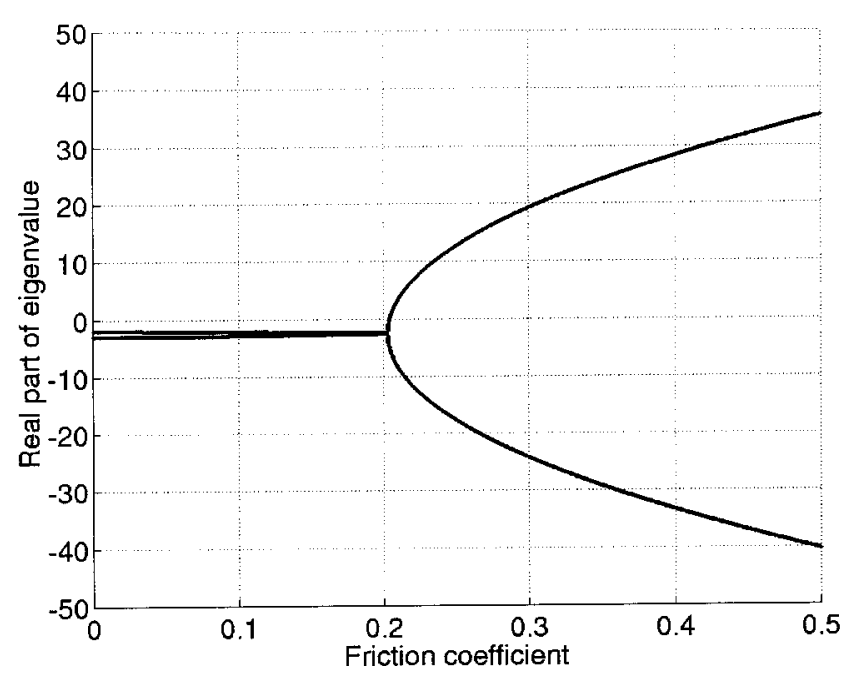

Fig. 3 Evolution of the real part of two coupling modes

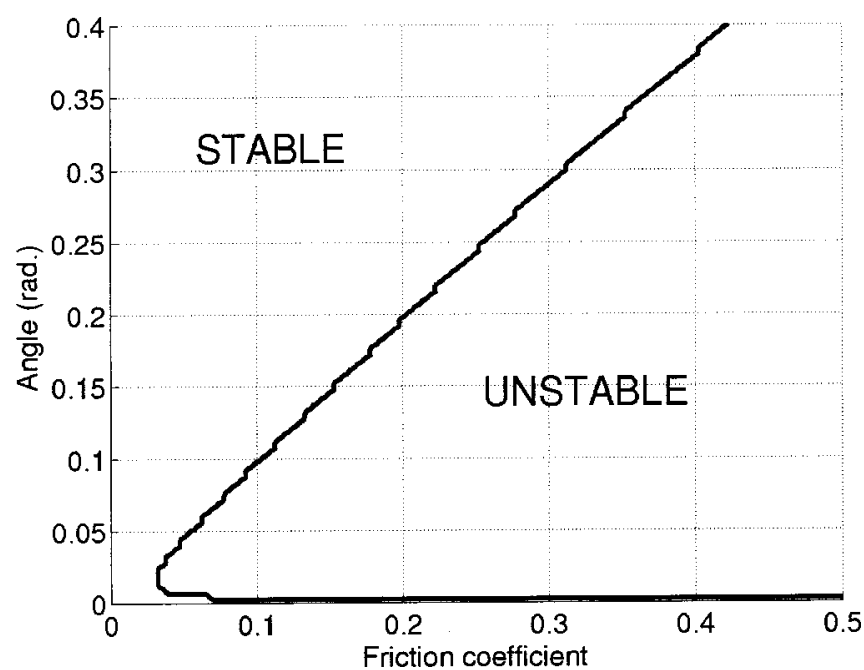

Fig. 4 Stability analysis as a function of the brake friction coefficient and the sprag-slip angle

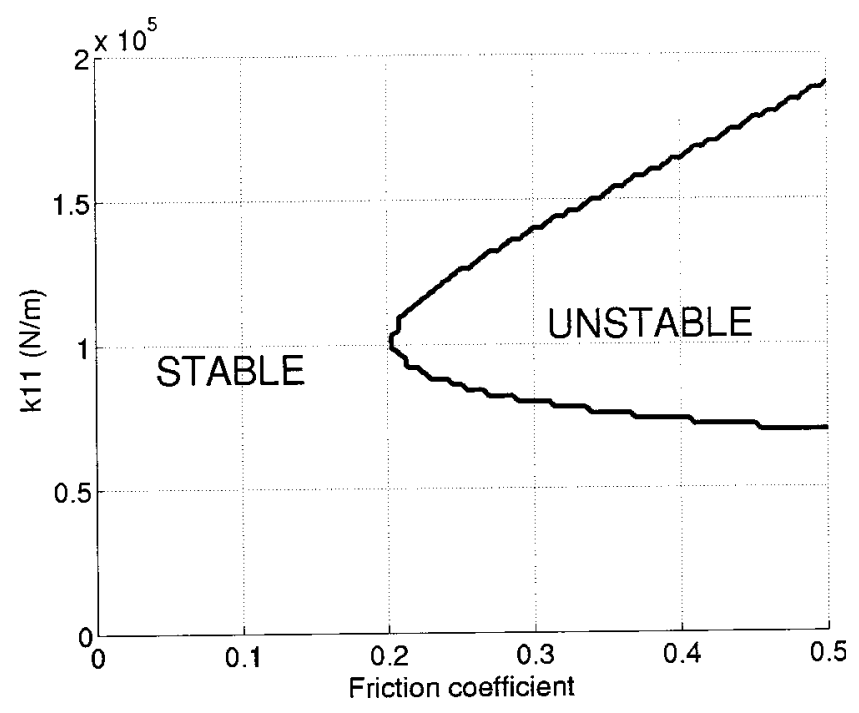

Fig. 5 Stability analysis as a function of the brake friction coefficient and the stiffness $k_{11}$

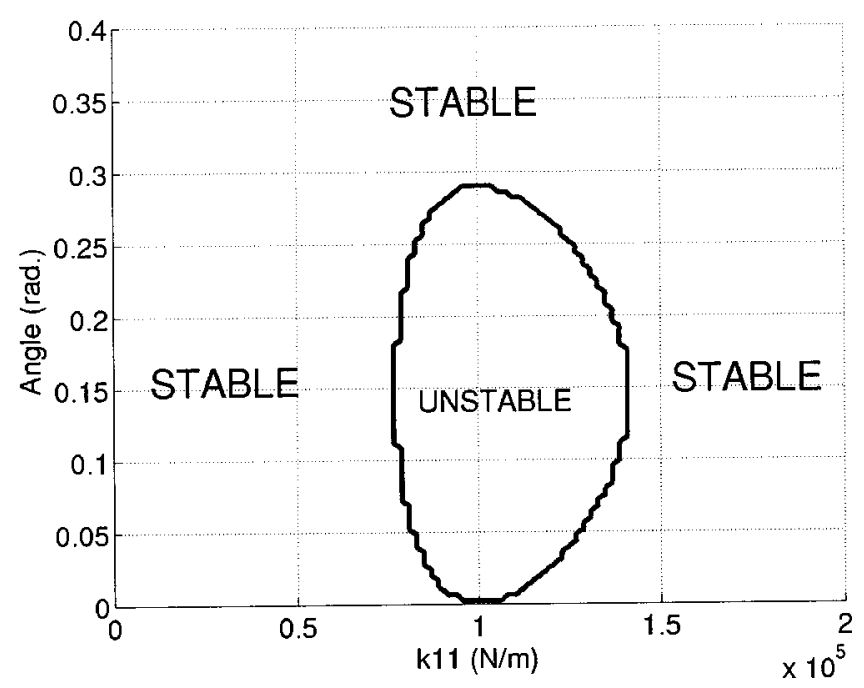

Fig. 6 Stability analysis as a function of the stiffness $k_{11}$ and the sprag-slip angle

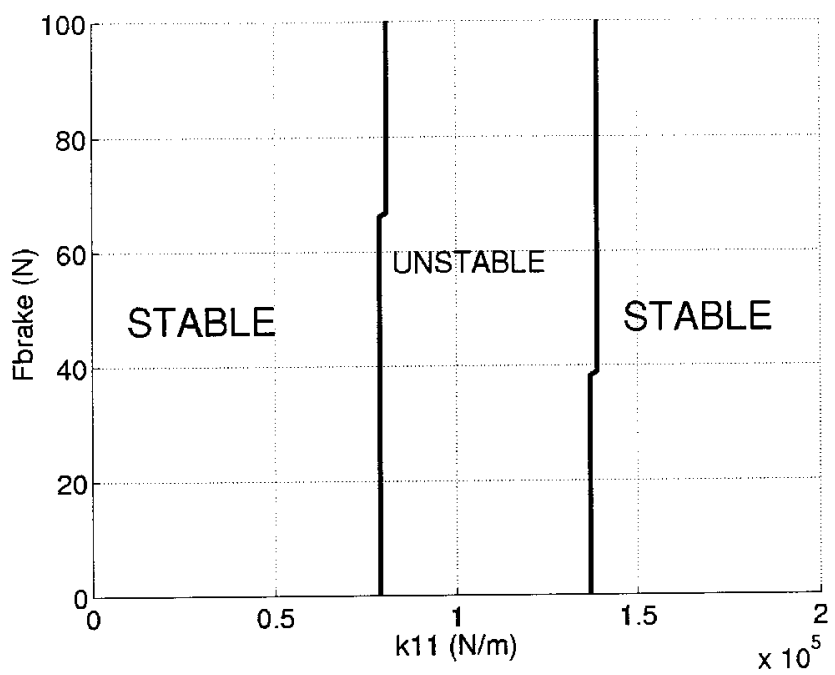

Fig. 7 Stability analysis as a function of the braking force $F_{\text {brake }}$ and the stiffness $k_{11}$ 


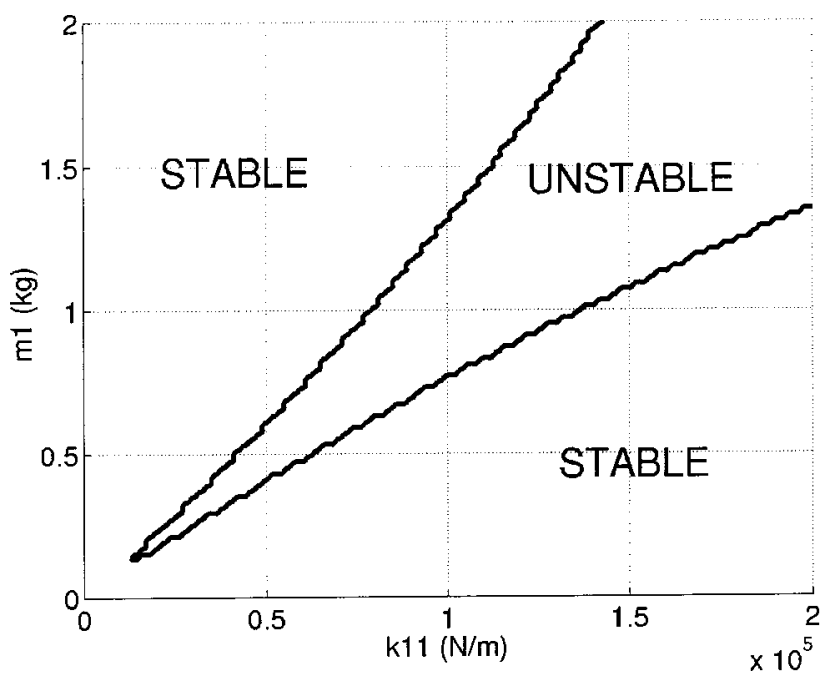

Fig. 8 Stability analysis as a function of the mass $m_{1}$ and the stiffness $k_{11}$

Fig. 2 and in Fig. 3, respectively. The system is unstable for $\mu$ $>\mu_{0}$ and stable for $\mu<\mu_{0}$. Moreover, the frequency $\omega_{0}$ of the unstable mode obtained for $\mu=\mu_{0}$ is near $50 \mathrm{~Hz}$. There is a perfect correlation with experiment tests where grabbing vibration is in the $40-70 \mathrm{~Hz}$ range.

Moreover, it is possible to perform a stability analysis using two parameters. The evolutions of stable and unstable regions versus two specific parameters are shown in Figs. 4-9. It is observed that stability is a complex problem. Parametric design studies show that stability can be altered by changes in the brake friction, stiffness, angle and mass. The most important design parameters are the brake friction coefficient, the linear stiffness $k_{11}$ and the angle $\theta$. To put it more precisely, decreasing brake friction coefficient reduces the unstable region, as illustrated in Figs. 4 and 5 . This is one way to stabilize the brake system. The angle $\theta$ is also important in the stabilization of the system, as shown in Fig. 4 and in Fig. 6. Moreover, increasing or decreasing stiffness and mass have some effect on the stable region, as illustrated in Figs. 4-7 and Fig. 9; decreasing both linear stiffness $k_{11}$ and mass $m_{1}$ reduces the unstable region, as indicated in Fig. 9. Therefore, one

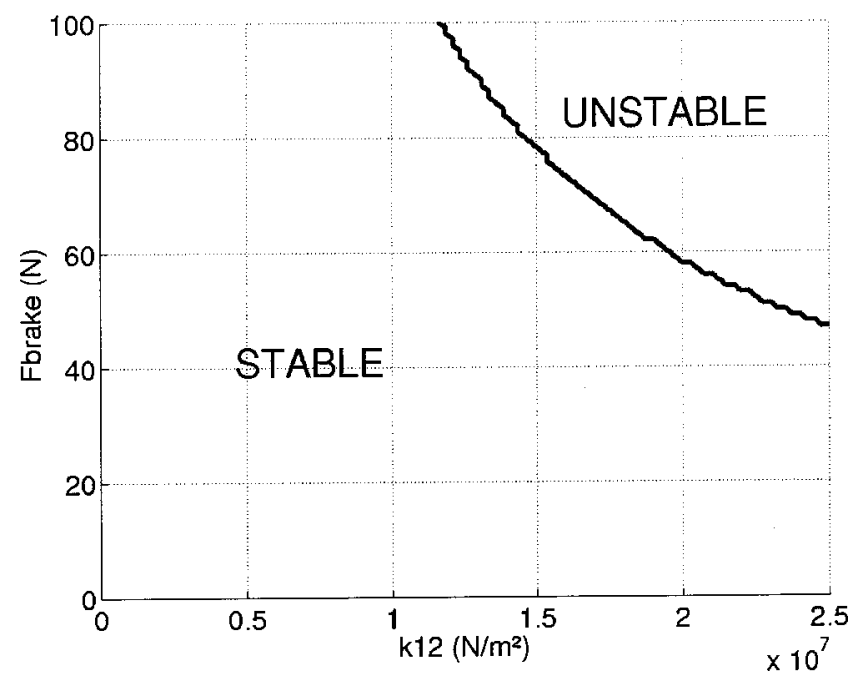

Fig. 9 Stability analysis as a function of the braking force $F_{\text {brake }}$ and the stiffness $k_{12}$ notes that the nonlinear stiffness $k_{12}$ and the brake force $F_{\text {brake }}$ have only small influences on the stability of the brake system, as shown in Figs. 7 and 8.

\section{Complex Nonlinear Problem}

The linear stability analysis of the preceding section gives the condition of stability. In the unstable region, any small disturbance will cause the motion to diverge from steady state equilibrium. In order to conduct a complex nonlinear analysis and to study the behavior in the unstable region, it is necessary to consider complete expressions of the nonlinear forces. The complete nonlinear expressions of the nonlinear forces are expressed at the equilibrium point $\mathbf{x}_{\mathbf{0}}$ for small perturbations $\overline{\mathbf{x}}$. One has $\mathbf{x}=\mathbf{x}_{\mathbf{0}}$ $+\overline{\mathbf{x}}$. The complete non-linear equations can be written as follow:

$$
\mathbf{M} . \ddot{\mathbf{x}}+\mathbf{C} \cdot \dot{\overline{\mathbf{x}}}+\mathbf{K} \cdot \overline{\mathbf{x}}=\mathbf{P}_{\mathrm{NL}}(\overline{\mathbf{x}})
$$

where $\ddot{\overline{\mathbf{x}}}, \dot{\overline{\mathbf{x}}}$ and $\overline{\mathbf{x}}$ are the acceleration, velocity, and displacement response two-dimensional vectors of the degrees-of-freedom, respectively. $\mathbf{M}$ is the mass matrix, $\mathbf{C}$ is the damping matrix and $\mathbf{K}$ is the stiffness matrix. $\mathbf{P}_{\mathbf{N L}}$ is the nonlinear force due to net brake hydraulic pressure and nonlinear stiffness. It contains the linear and nonlinear terms about the equilibrium point for small perturbations.

The nonlinear sprag-slip equation about the equilibrium point $\mathbf{x}_{\mathbf{0}}=\left\{\begin{array}{ll}X_{0} & Y_{0}\end{array}\right\}^{T}$ for small perturbations $\overline{\mathbf{x}}=\{\bar{X} \bar{Y}\}^{T}$ can be expressed as

$\mathbf{M} . \ddot{\mathbf{x}}+\mathbf{C} \cdot \dot{\dot{\mathbf{x}}}+\widetilde{\mathbf{K}} \cdot \overline{\mathbf{x}}=\sum_{i=1}^{2} \sum_{j=1}^{2} \mathbf{f}_{(\mathbf{2})}^{\mathrm{ij}} \cdot \bar{x}_{j} \cdot \bar{x}_{j}+\sum_{i=1}^{2} \sum_{j=1}^{2} \sum_{k=1}^{2} \mathbf{f}_{(\mathbf{3})}^{\mathrm{ijk}} \cdot \bar{x}_{i} \cdot \bar{x}_{j} \cdot \bar{x}_{k}$

One notes that $\widetilde{\mathbf{K}}$ is the stiffness matrix containing the terms of the first stiffness matrix $\mathbf{K}$ and the linear terms of $\mathbf{P}_{\mathrm{NL}}$ at the equilibrium point. The vectors $\mathbf{f}_{(\mathbf{2})}^{\mathbf{i j}}$ and $\mathbf{f}_{(\mathbf{3})}^{\mathbf{i j k}}$ are the coefficients of the quadratic and cubic terms, respectively, due to the non-linear stiffness about the equilibrium point. In order to obtain time-history responses, the complete set of nonlinear dynamic equations has to be integrated numerically. However this procedure is time consuming, when parametric design studies are needed. So the Complex Nonlinear Modal Analysis will be presented in order to obtain the limit cycle.

In order to use this new approach, the nonlinear equations are written with the state variables

$$
\begin{aligned}
& \dot{\mathbf{y}}=\mathbf{A} . \mathbf{y}+\sum_{i=1}^{4} \sum_{j=1}^{4} \boldsymbol{\eta}_{(\mathbf{2})}^{\mathbf{i j}} \cdot y_{i} \cdot y_{j}+\sum_{i=1}^{4} \sum_{j=1}^{4} \sum_{k=1}^{4} \boldsymbol{\eta}_{(\mathbf{3})}^{\mathbf{i j k}} \cdot y_{i} \cdot y_{j} \cdot y_{k} \\
& \text { where } \mathbf{y}=\left\{\overline{\mathbf{x}} \dot{\overline{\mathbf{x}}}^{T}, \quad \mathbf{A}=-\left[\begin{array}{cc}
\mathbf{C} & \mathbf{M} \\
\mathbf{I} & \mathbf{0}
\end{array}\right]^{-1} \cdot\left[\begin{array}{cc}
\widetilde{\mathbf{K}} & \mathbf{0} \\
\mathbf{0} & \mathbf{I}
\end{array}\right],\right. \\
& \boldsymbol{\eta}_{(\mathbf{2})}=\left[\begin{array}{cc}
\mathbf{C} & \mathbf{M} \\
\mathbf{I} & \mathbf{0}
\end{array}\right]^{-1} \cdot\left[\begin{array}{c}
\mathbf{f}_{(\mathbf{2})} \\
\mathbf{0}
\end{array}\right], \quad \text { and } \quad \boldsymbol{\eta}_{(\mathbf{3})}=\left[\begin{array}{cc}
\mathbf{C} & \mathbf{M} \\
\mathbf{I} & \mathbf{0}
\end{array}\right]^{-1} \cdot\left[\begin{array}{c}
\mathbf{f}_{(\mathbf{3})} \\
\mathbf{0}
\end{array}\right] .
\end{aligned}
$$

$\boldsymbol{\eta}_{(\mathbf{2})}^{\mathrm{ij}}$ and $\boldsymbol{\eta}_{(\mathbf{3})}^{\mathrm{ijk}}$ are quadratic and cubic non-linear terms of the state variables, respectively.

Finally, the problem can be put into Jordan normal form by means of the eigenbasis. Since, one considers here the physically interesting case of the stable equilibrium losing stability; the previous system can be written in the form

$$
\dot{\mathbf{z}}=\mathbf{J . z}+\mathbf{F N L}(\mathbf{z})
$$

where $\mathbf{J}$ is the linear matrix and $\mathbf{F N L}(\mathbf{z})$ defined the vector of the non-linear terms. It may be observed that the steps involved in transforming the nonlinear second order equations as expressed in 
physical coordinates into a first-order Jordan form can be computationally demanding, especially if many degrees of freedom are involved (Sridhar and Jordan [24]).

\section{The Method of Equivalent Linearization}

In order to obtain the one-periodic response of a nonlinear system, it is possible to replace the nonlinear system by an equivalent linear system. This method is based on the well-known technique of equivalent linearization of Kryloff and Bogoliubov [25]. The principle of this method to substitute the nonlinear system by a linear system. This associated linear system is sought such that the difference between the two systems is minimized. Then, the solution of the associated linear system is taken as an approximation of the original problem. Iwan [19] extended the method of equivalent linearization to apply to multi-degree-of-freedom systems. Several questions, pertaining to the existence and uniqueness, have been examined by Spanos and Iwan [26-28].

One considers the nonlinear differential equation (9) with the non-linear vector function $\mathbf{F N L}(\mathbf{z})$. The equivalent linear system may be expressed in the form

$$
\dot{\tilde{\mathbf{z}}}=\mathbf{J} \widetilde{\mathbf{z}}+\mathbf{J}^{\prime} \widetilde{\mathbf{z}}
$$

$\mathbf{J}^{\prime}$ is the equivalent linear matrix. It is obtained by minimizing the difference $\boldsymbol{\varepsilon}$ between the nonlinear equation (9) and the equivalent linear equation (10) for every $\widetilde{\mathbf{z}}(\mathbf{t})$, where $\boldsymbol{\varepsilon}$ is the difference

$$
\boldsymbol{\varepsilon}=\mathbf{F N L}(\widetilde{\mathbf{z}})-\mathbf{J}^{\prime}(\widetilde{\mathbf{z}})
$$

The minimization of $\boldsymbol{\varepsilon}$ is performed according to the criterion $\operatorname{Min}\left(\int_{0}^{2 \pi / \omega} \boldsymbol{\varepsilon}^{T} \boldsymbol{\varepsilon}\right)$. The method used to solve the optimization problem is the least-square method. In place of the mean square criterion specified previously, other criterion can be used as well. However, a comparative study of the most commonly used criteria has shown that there is no significant superiority in terms of approximate solution accuracy of any specific criterion over the others (Iwan [27]).

\section{The Complex Nonlinear Modal Analysis}

One has to consider a unstable nonlinear autonomous system. One supposes that the eigenvalues of the linearized system at the equilibrium point are complex, one having a positive real part, and the other a negative one. One can consider that all the modal participations of the modes are then negligible in front of the unstable mode. The unstable solution curve of the system can be simply written while utilizing only this unstable mode: $\widetilde{\mathbf{z}}(t)$ $=\mathbf{Z}_{0} e^{\lambda t}+\overline{\mathbf{Z}_{0}} e^{-\lambda t} . \lambda$ is the eigenvalue having a positive real part and $\mathbf{Z}_{\mathbf{0}}$ is the eigenvector corresponding to the eigenvalue $\lambda$. The real part of the eigenvalue $\lambda$ then characterizes the stability of the system and the imaginary part corresponds to the frequency of the associated instability.

Next, one considers the stable periodic solution of the equivalent linearized system; the modal participations of the stable modes are considered negligible compared to the modal participation of the unstable mode. So this stable periodic solution can be written as follow

$$
\widetilde{\mathbf{Z}}(t)=p\left(\mathbf{Z}_{\mathbf{0}}(p) e^{i \omega(p) t}+\overline{\mathbf{Z}_{\mathbf{0}}(p)} e^{-i \omega(p) t}\right)
$$

where $p$ defines the amplitude of the stationary periodic solution of the system, $\omega(p)$ and $\mathbf{Z}_{0}(p)$ the associated frequency and eigenvector, respectively.

Now, in order to obtain the value of $p, \omega(p)$ and $\mathbf{Z}_{0}(p)$, one considers the eigenvalue $\lambda$ and the eigenvector $\mathbf{Z}_{\mathbf{0}}$ of the unstable mode for $p=0$. This eigenvalue and this eigenvector are determined by considering the linearized system at the equilibrium point. One notices that for $p=0$, the eigenvalue $\lambda$ has a positive real part. The objective is to be able to follow the evolution of the

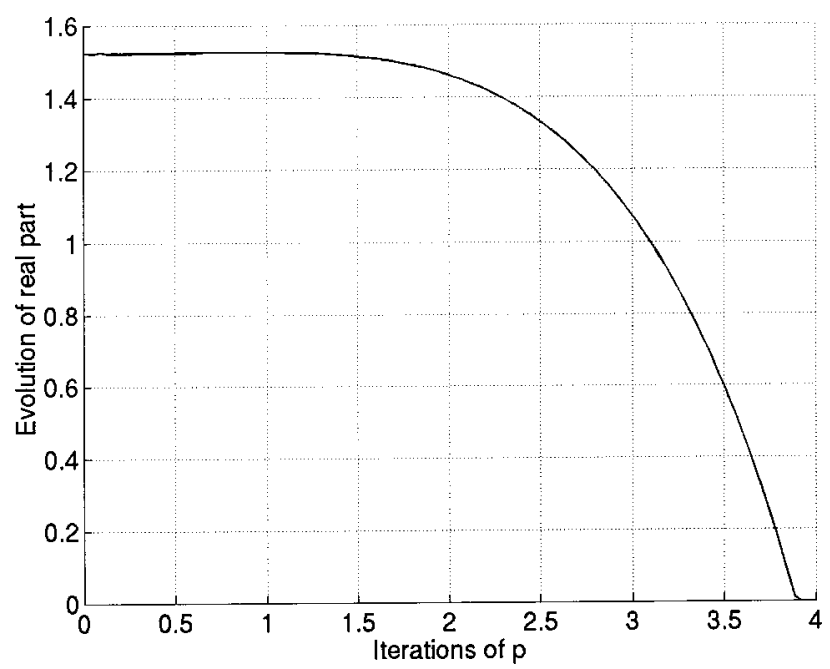

Fig. 10 Evolution of the real part of the unstable eigenvalue

eigenvalue $\lambda(p)$ and the associated deformation $\mathbf{Z}_{\mathbf{0}}(p)$ that lead to the stationary periodic solution of the system; these values will be obtained when $\operatorname{Re}[\lambda(p)]=0$.

By incrementing the value $p=p+\delta p$ and by considering the equivalente linearized system (29) of the nonlinear system (28) for every $\widetilde{\mathbf{z}}(t)=p\left(\mathbf{Z}_{\mathbf{0}}(p) e^{i \omega(p) t}+\overline{\mathbf{Z}_{\mathbf{0}}(p)} e^{-i \omega(p) t}\right)$, obtained by using the concept of equivalent linearization, one obtains a new linearized system. The previous eigenvalue $\lambda(p)$ becomes $\lambda(p$ $+\delta p)$ and the associated eigenvector $\mathbf{Z}_{\mathbf{0}}(p+\delta p)$. The real part of the eigenvalue $\lambda(p)$ determined the stability of the equivalent linearized system for the amplitude $p$. If the eigenvalues of the linearized system at the equilibrium point are complex, one having null real part and the other negative real part, the system, the amplitude $p$ corresponds to the amplitude of the stationary periodic solution of the non-linear system, and $\operatorname{Im}[\lambda(p)]$ is the frequency of the nonlinear system.

This method, called the Complex Nonlinear Modal Analysis (Boudot [11-12], Sinou [13]) is applied to obtain the limit cycles of the nonlinear equation (10). The evolution of the real part and the evolution of the nonlinear associated frequency are given in Figs. 10 and 11.

The stationary periodic solution of the system and the limit cycles are given Figs. 12 and 13. The limit cycles obtained by the

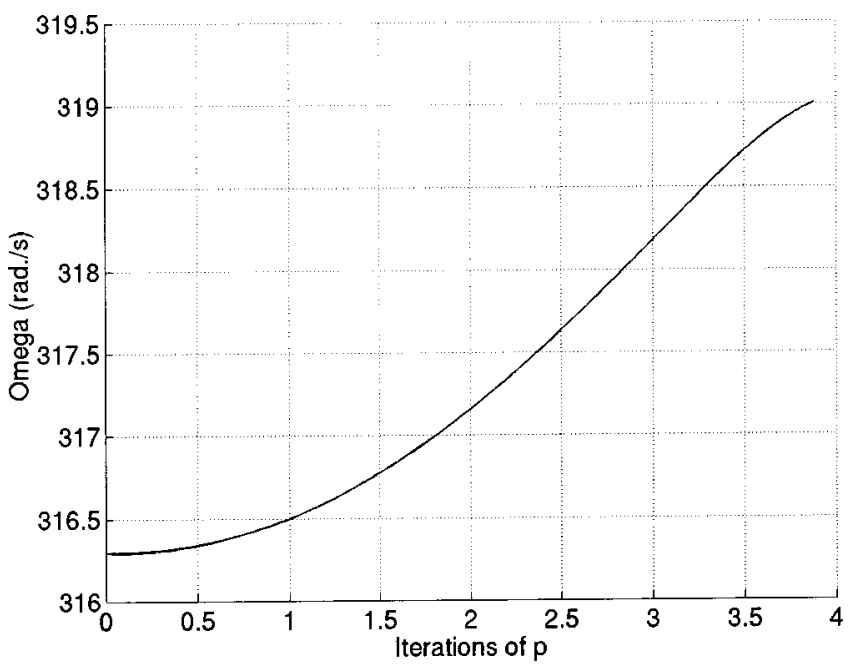

Fig. 11 Evolution of the imaginary part of the unstable eigenvalue 
CNLM-Analysis are compared with those obtained via the integration of the full nonlinear system.

Then, some indications have been observed by varying one parameter for the base values defined previously. It may be noted that the limit cycle is defined near the Hopf bifurcation point by using the brake friction coefficient as an unfolding parameter. It is observed that the level amplitude is a very complex problem. Indeed, the evolution of limit cycle amplitude is not linear with the evolution of specific parameter. Increasing or decreasing level amplitude versus linear evolution of a specific parameter can be observed. This is further reflected Figs. 14 and 15. Limit cycles increase and decrease with constant increasing of the angle $\theta$. Parametric studies of the evolution of limit cycles are a complex problem.

\section{Summary and Conclusion}

A nonlinear model for the analysis of mode heavy truck grabbing has been developed. The stability analysis indicates that system instability can occur with a constant friction coefficient. To further understanding of the effects of varying the parameters, stability analysis using two parameter evolutions has been rea-

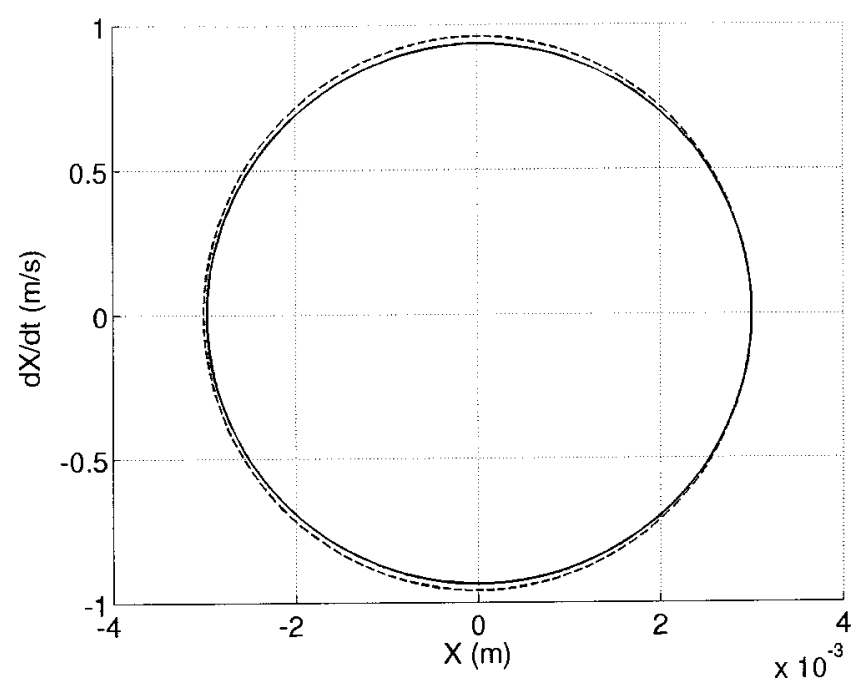

Fig. 12 X-limit cycle for $\bar{\mu}=\mu_{0} / 100$

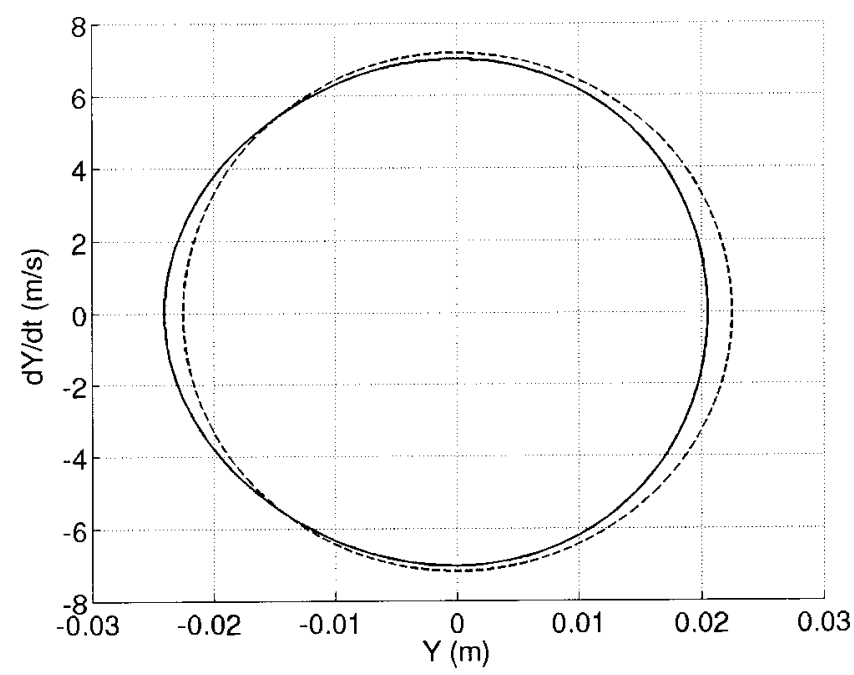

Fig. 13 Y-limit cycle for $\mu^{-}=\mu_{0} / 100$

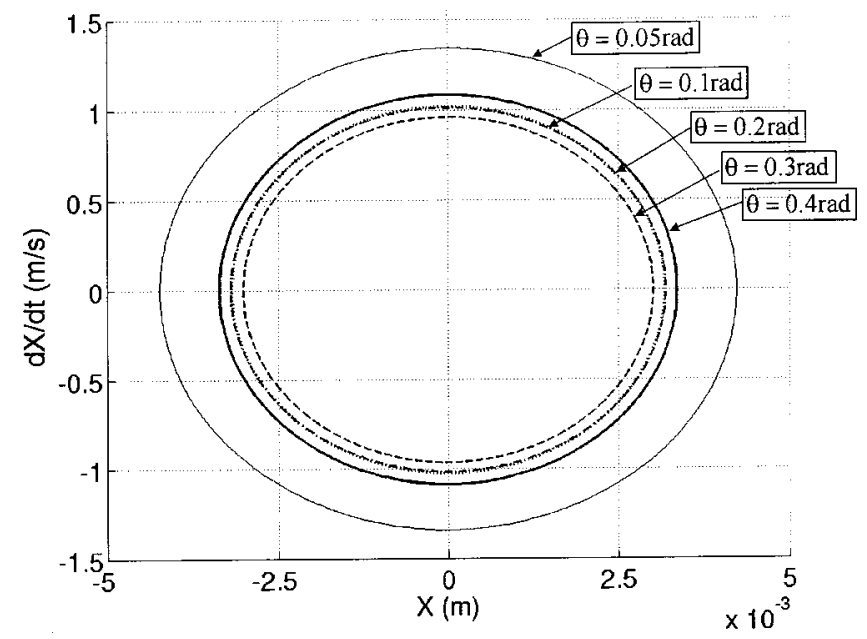

Fig. 14 X-limit cycle $\bar{\mu}=\mu_{0} / 100$ as a function of angle $\theta$

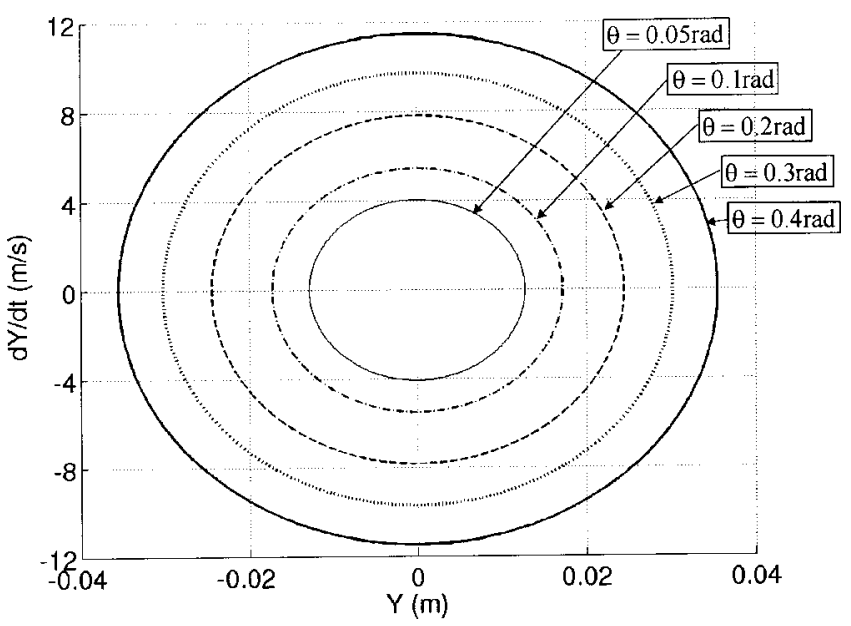

Fig. 15 Y-limit cycle $\bar{\mu}=\mu_{0} / 100$ as a function of angle $\theta$

lised. Changes in stiffness, brake friction coefficient, and angle of the sprag-slip phenomena may be significant on stability.

Moreover, this paper presents the Complex Nonlinear Modal Analysis in order to the limit cycle amplitudes. Excellent agreements are found between the results obtained by the CNLMAnalysis and the complete solution of the nonlinear system. However, the CNLM-Analysis is very simple and requires few computer resources. The CNLM-Analysis is very interesting when time history response solutions of the full set of nonlinear equations are time consuming to perform when extensive parametric design studies are needed.

\section{Acknowledgments}

The authors gratefully acknowledge the French Education Ministry for its support through grant No. 99071 for the investigation presented here.

\section{Nomenclature}

$$
\begin{aligned}
x & =\text { scalar } \\
\mathbf{x} & =\text { vector } \\
\dot{\mathbf{x}} & =\text { vector of velocity } \\
\ddot{\mathbf{x}} & =\text { vector of acceleration } \\
\mathbf{x}_{\mathbf{0}} & =\text { equilibrium point }
\end{aligned}
$$




$$
\begin{aligned}
\overline{\mathbf{x}} & =\text { small perturbation } \\
\mathbf{C} & =\text { damping matrix } \\
\mathbf{K} & =\text { stiffness matrix } \\
\mathbf{M} & =\text { mass matrix } \\
\mathbf{J} & =\text { Jacobian matrix of the system } \\
\mathbf{F} & =\text { vector force } \\
\mathbf{P}_{\mathbf{N L}} & =\text { vector of linear and nonlinear terms } \\
\theta & =\text { sprag-slip angle } \\
\mu & =\text { brake friction coefficient } \\
\mu_{0} & =\text { brake friction coefficient at the Hopf bifurcation point } \\
\mathbf{f}_{(\mathbf{2})}^{\mathrm{ij}} & =\text { coefficients of quadratic nonlinear terms } \\
\mathbf{f}_{(\mathbf{3})}^{\mathbf{i j k}} & =\text { coefficients of cubic nonlinear terms } \\
\boldsymbol{\eta}_{(\mathbf{2})} & =\text { coefficients of quadratic nonlinear terms in state vari- } \\
& \text { ables } \\
\boldsymbol{\eta}_{(\mathbf{3})}^{\mathrm{jik}}= & \text { coefficients of cubic nonlinear terms in state variables }
\end{aligned}
$$

\section{Appendix A: Parameter Values}

$$
\begin{aligned}
F_{\text {brake }}=1 \mathrm{~N} & =\text { brake force } \\
m_{1}=1 \mathrm{~kg} & =\text { equivalent mass of first mode } \\
m_{2}=1 \mathrm{~kg} & =\text { equivalent mass of second mode } \\
c_{1}=5 \mathrm{~N} / \mathrm{m} / \mathrm{sec}= & \text { equivalent damping of first mode } \\
c_{2}=5 \mathrm{~N} / \mathrm{m} / \mathrm{sec}= & \text { equivalent damping of second mode } \\
k_{11}=1.10^{5} \mathrm{~N} / \mathrm{m}= & \text { coefficient of linear term of stiffness } k_{1} \\
k_{12}=1.10^{6} \mathrm{~N} / \mathrm{m}^{2}= & \text { coefficient of quadratic term of } \\
& \text { stiffness } k_{1} \\
k_{13}=1.10^{6} \mathrm{~N} / \mathrm{m}^{3}= & \text { coefficient of cubic term of stiffness } k_{1} \\
k_{21}=1.10^{5} \mathrm{~N} / \mathrm{m}^{2}= & \text { coefficient of linear term of stiffness } k_{2} \\
k_{22}=1.10^{5} \mathrm{~N} / \mathrm{m}^{2}= & \text { coefficient of quadratic term of } \\
& \text { stiffness } k_{2} \\
k_{23}=1.10^{5} \mathrm{~N} / \mathrm{m}^{3}= & \text { coefficient of cubic term of stiffness } k_{2} \\
\theta=0,2 \mathrm{rad}= & \text { sprag-slip angle }
\end{aligned}
$$

\section{Appendix B: Values of $\mathrm{M}, \mathrm{C}, \mathrm{K}, \mathrm{F}$ and $\mathrm{F}_{\mathrm{NL}}$}

The expressions of the matrices $\mathbf{M}, \mathbf{C}, \mathbf{K}, \mathbf{F}$ and $\mathbf{F}_{\mathbf{N L}}$ are

$$
\begin{aligned}
& \mathbf{M}=\left[\begin{array}{cc}
m_{2}\left(\tan ^{2} \theta+1\right) & 0 \\
0 & m_{1}
\end{array}\right], \\
& \mathbf{C}=\left[\begin{array}{cc}
c_{1}\left(\tan ^{2} \theta-\mu \tan \theta\right)+c_{2}(1+\mu \tan \theta) & c_{1}(-\tan \theta+\mu) \\
-c_{1} \tan \theta & c_{1}
\end{array}\right] \\
& \mathbf{K}=\left[\begin{array}{cc}
k_{21}(1+\mu \tan \theta)+k_{11}\left(\tan ^{2} \theta-\mu \tan \theta\right) & k_{11}(-\tan \theta+\mu) \\
-k_{11} \tan \theta & k_{11}
\end{array}\right], \\
& \left.\mathbf{F}=\left\{\begin{array}{c}
0 \\
-F_{\text {brake }}
\end{array}\right\} \begin{array}{c}
(-\tan \theta+\mu)\left(k_{12}(X \tan \theta-Y)^{2}+k_{13}(X \tan \theta-Y)^{3}\right) \\
+k_{22}(1+\mu \tan \theta) X^{2}+k_{23}(1+\mu \tan \theta) X^{3} \\
-k_{12}(Y-X \tan \theta)^{2}-k_{13}(Y-X \tan \theta)^{3}
\end{array}\right\}
\end{aligned}
$$

\section{References}

[1] Pierre, C., Ferri, A. A., and Dowell, E. H., 1985, "Multi-Harmonic Analysis of Dry Friction Damped Systems Using an Incremental Harmonic Balance Method," ASME J. Appl. Mech., 52, pp. 958-964.

[2] Cameron, T. M., and Griffin, J. H., 1989, "An Alternating Frequency/Time Domain Method for Calculating the Steady State Response of Nonlinear Dynamic," ASME J. Appl. Mech., 56, pp. 149-154.

[3] Nayfeh, A. H., and Mook, D. T., 1979, Nonlinear Oscillations, John Wiley \& Sons, New-York.

[4] Nayfeh, A. H., and Balachandran, B., 1995, Applied Nonlinear Dynamics: Analytical, Computational and Experimental Methods, John Wiley \& Sons.

[5] Guckenheimer, J., and Holmes, P., 1986, Nonlinear Oscillations, Dynamical Systems, and Bifurcations of Vector Fields, Springer-Verlag.

[6] Jezequel, L., and Lamarque, C. H., 1991, "Analysis of Non-linear Dynamical Systems by the Normal Form Theory," J. Sound Vib., 149, pp. 429-459.

[7] Yu, P., 1998, "Computation of Normal Forms via a Perturbation Technique," J. Sound Vib., 211, pp. 19-38.

[8] Sinou, J. J., Thouverez, F., and Jézéquel, L., 2003, "Center Manifold and Multivariable Approximants Applied to Non-Linear Stability Analysis," Int. J. Non-Linear Mech., 38/9, pp. 1421-1442.

[9] Baker, G. A., and Graves-Morris, P., 1996, Padé Approximants, Cambridge University Press, Cambridge.

[10] Brezinski, C., 1983, "Extrapolation Algorithms and Padé Approximations: A Historical Survey," Applied Numerical Mathematics, 20, pp. 299-318.

[11] Boudot, J. P., Carneiro, A., and Jézéquel, L., 1998, “Analyze des phénomènes intervenant dans la stabilité des systèmes de freinage. Influence des nonlinéarités," JEF95, Journées Européennes de Freinage, Edition AGIR, Lille, 1, pp. 213-224

[12] Boudot, J. P., 1995, "Modélisation des bruits de freinage des véhicules Industriels," Thèse de Doctorat, No. 1995-08, Ecole Centrale de Lyon, Ecully.

[13] Sinou, J-J., 2002, "Synthèse non-linéaire des systèmes vibrants. Application aux systèmes de freinage," Thèse de Doctorat, No. 2002-23, Ecole Centrale de Lyon, Ecully.

[14] Rosenberg, R. M., 1962, "The Normal Modes of Nonlinear n-Degree-ofFreedom Systems,” ASME J. Appl. Mech., 82, pp. 7-14.

[15] Rosenberg, R. M., 1966, "On Non-Linear Vibrations of Systems with Many Degree of Freedom," Adv. Appl. Mech., 9, pp. 155-242.

[16] Szemplinska-Stupinicka, W., 1979, "The Modified Single Mode Method in the Investigation of the Resonant Vibration of Non-Linear Systems," J. Sound Vib., 104(2), pp. 475-489.

[17] Szemplinska-Stupinicka, W., 1990, The Behavior of Nonlinear Vibrating Systems, Kluwer Academic.

[18] Shaw, S. W., and Pierre, C., 1993, "Normal Modes for Non-Linear Vibratory Systems," J. Sound Vib., 164(1), pp. 85-124.

[19] Iwan, W. D., 1973, “A Generalization of the Concept of Equivalent Linearization," Int. J. Non-Linear Mech., 8, pp. 279-287.

[20] Ibrahim, R. A., 1994, "Friction-Induced Vibration, Chatter, Squeal and Chaos: Part II-Dynamics and Modeling," ASME J. Appl. Mech., 47(7), pp. 227-253.

[21] Oden, J. T., and Martins, J. A. C., 1985, "Models and Computational Methods for Dynamic friction Phenomena," Comput. Methods Appl. Mech. Eng., 52, pp. 527-634.

[22] Crolla, D. A., and Lang, A. M., "Brake Noise and Vibration-State of Art," Tribologie Series 18, Vehicle Tribology, Paper VII, pp. 165-174, Dowson, Taylor \& Godet, eds., Elsevier.

[23] Spurr, R. T., 1961-1962, “A Theory of Brake Squeal,” Proc. Inst. Mech. Eng., Part D (J. Automob. Eng.), 1, pp. 33-40.

[24] Sridhar, B., and Jordan, D., 1973, "An Algorithm for Calculation of the Jordan Canonical Form of a Matrix," Comput. \& Elect. Engng, 1, pp. 239-254.

[25] Bogoliubov, N., and Mitropolski, A., 1961, Asymptotic Methods in the Theory of Nonlinear Oscillations, Gordon and Breach, New York.

[26] Spanos, P-T. D., and Iwan, W. D., 1978, "On the Existence and Uniqueness of Solutions Generated by Equivalent Linearization," Int. J. Non-Linear Mech. 13, pp. 71-78.

[27] Iwan, W. D., and Patula, F. J., 1972, "The Merit of Different Minimization Criteria in Approximate Analysis," ASME J. Appl. Mech., 39, pp. 257-262.

[28] Spanos, P-T. D., and Iwan, W. D., 1979, "Harmonic Analysis of Dynamic Systems with Nonsymmetric Nonlinearities," ASME J. Dyn. Syst., Meas. Control, 101, pp. 31-36. 